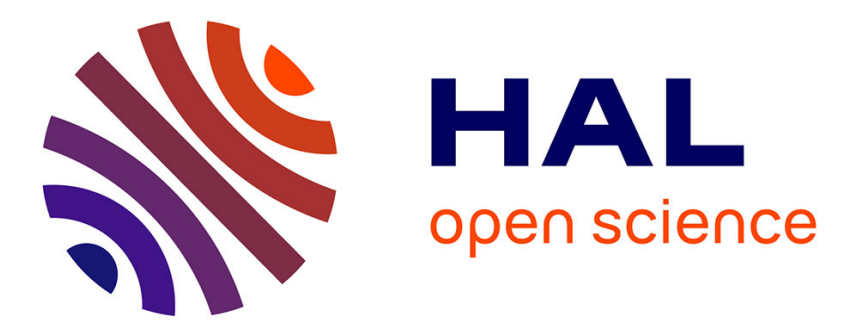

\title{
Probabilistic Error Model for a Lane Marking Based Vehicle Localization Coupled to Open Source Maps
}

Wenjie Lu, Sergio Alberto Rodriguez Florez, Emmanuel Seignez, Roger Reynaud

\section{- To cite this version:}

Wenjie Lu, Sergio Alberto Rodriguez Florez, Emmanuel Seignez, Roger Reynaud. Probabilistic Error Model for a Lane Marking Based Vehicle Localization Coupled to Open Source Maps. 2014 IEEE 17th International Conference on Intelligent Transportation Systems (ITSC), Oct 2014, Qingdao, China. hal-01075839

\section{HAL Id: hal-01075839 \\ https://hal.science/hal-01075839}

Submitted on 20 Oct 2014

HAL is a multi-disciplinary open access archive for the deposit and dissemination of scientific research documents, whether they are published or not. The documents may come from teaching and research institutions in France or abroad, or from public or private research centers.
L'archive ouverte pluridisciplinaire HAL, est destinée au dépôt et à la diffusion de documents scientifiques de niveau recherche, publiés ou non, émanant des établissements d'enseignement et de recherche français ou étrangers, des laboratoires publics ou privés. 


\title{
Probabilistic Error Model for a Lane Marking Based Vehicle Localization Coupled to Open Source Maps
}

\author{
Wenjie Lu ${ }^{1,2}$, Sergio A. Rodriguez F. ${ }^{1,2}$, Emmanuel Seignez ${ }^{1,2}$ and Roger Reynaud ${ }^{1,2}$
}

\begin{abstract}
Recent works have focused on lane marking feature based vehicle localization using enriched maps. The localization precision of existing methods depends strongly on the accuracy of the maps which are specially customized. In this article, we propose a marking feature based vehicle localization using open source map. Our method makes use of multi-criterion confidences to infer potential errors, and in advance, to enhance the vehicle localization. At first, the vision-based lane marking models are obtained. Meanwhile, the map-based lane markings of current state are derived from map databases. Both lane marking sources are fused together to implement vehicle localization, using a multi-kernel based algorithm. In order to further improve the localization performance, a probabilistic error model is employed to identify the possible errors. The experiments have been carried out on public database. The results show that error modeling leads to a lower average lateral error in localization result.
\end{abstract}

\section{INTRODUCTION}

Map-matching algorithms integrate the positioning data from GPS sensors with Geographic Information Systems (GIS). The algorithms identify the current road section on which the host vehicle is traveling, then determine the position of the host vehicle on the identified road section. The purpose of a map-matching method is to enhance the localization performance, and in advance to improve navigation function in Intelligent Transport Systems (ITS). Stateof-the-art map-matching methods can be classified into four categories: geometric approaches, topological approaches, probabilistic approaches, and advanced approaches.

Geometric approaches. A geometric approach takes only the road section shapes into account, without the connection relationships of different links. The techniques of this category include matching a single vehicle position to a single map point [1], a single vehicle position to a map road curve [2], and a vehicle trajectory curve to a map road curve [3]. Methods in this category own high real-time ability because these techniques have low complexity. However, geometric approaches are sensible to noises.

Topological approaches. Topological approaches are able to solve the noise sensitivity problem occurred in geometric means. The idea is to introduce topological information of map to help to reduce the possible road curve candidates. In [4], an improved curve to curve matching algorithm is demonstrated. Several criteria are used to help identify the current road section, with the vehicle trajectory from GPS. Differences between vehicle bearing and road direction are

1. Université Paris-Sud, 91405, Orsay, FRANCE

2. Institut Fondamentale d'Electronique CNRS 8622, 91405, Orsay, FRANCE computed, as well as distance to road segment, to determine the current road section. At road junctions, an algorithm is designed to decide which branch the vehicle drives to, considering connectivity, legal possible turn, and difference of vehicle bearing change and road direction changes.

Probabilistic approaches. Instead of a specific estimated location, an elliptical or rectangular confidence region is provided using probabilistic methods [5]. In [6], a confidence region is created according to Dead Reckoning position. This confidence region is computed only in complex conditions, such as junctions, reducing the algorithm executing time. In [7], a probabilistic value is derived from the structure tensor of camera images. This value is used to match camera images and images created from the map database.

Advanced approaches. In recent years, advanced algorithms and methods are employed on map matching problem. On the one hand, algorithms with refined concepts are introduced. Common methods in vehicle localization include Kalman Filter [8], Extended Kalman Filter [9], optimization algorithm [10], and methods relying on interval analysis [11]. On the other hand, various cues are used to enhance map matching results. In [12], objects localization is regarded as one criterion to assist vehicle localization. In [13], traffic signs (i.e. arrows, pedestrian crossings and markings) from both vision source and map source are compared to provide a transformation from vision space to map space.

Localization using lane marking is considered as one branch of map-matching localization. Publications as [13][14] on this direction utilize high-accuracy digital maps, including the precise positions of the markings. In [13], a translation vector between vision space and map space are derived according to traffic signs (i.e. markings and pedestrian crossings) from both sources, which helps adjust vehicle location. High-accuracy digital maps help improve localization results. However, these maps are specially customized, which is time-consuming and range-limited. Some papers use normal map database. In [15], a map of lane marking features is built. Vehicle localization is implemented using this map and vision based marking features.

In this paper, a lane marking feature based vehicle localization method is proposed. The aim is to estimate accurate vehicle localization coupling vision information and open source map database. In addition, the performance of vehicle localization is improved, taking probabilistic error model into account. In Section II, lane markings are detected through image processing. In Section III, vehicle location is estimated according to fusing both vision-based lane markings and markings from reconstructed map databases. Section 
IV designs an error model to search for errors in vehicle localization method. Finally, Section V presents experimental results.

\section{LANE MARKING DETECTION}

Road marking featuresprovided by camera images are extracted through image processing. The marking model parameters are then estimated using an improved multi-kernel method. The lane marking detection algorithm is proposed in [16].

The image processing procedure includes the following steps. Inverse Perspective Mapping (IPM) translation transmits perspective space images into Bird Eye's View (BEV) space image, which greatly facilitates marking detection strategies. A second order derivative filter along the horizontal direction is applied to process BEV images where lane markings are nearly vertical. To eliminate the remaining outliers, a cell-based blob algorithm, improved from [17], is introduced. At the end of image processing part, a binary image representing lane marking pixel candidates is obtained.

A parabola in BEV space: $x=c+d \cdot y+e \cdot y^{2}$ is selected as marking model. The marking initialization step determines the zero order component $c$. The other two components $d$ and $e$ are estimated through a multi-kernel based method.

\section{LANE MARKING BASED VEHICLE LOCALIZATION}

\section{A. Particle Filter}

A particle filter is designed to estimate vehicle positions in East North Up (ENU) space using the derived marking models. The observation of the filter is rough GPS signal. The state of a particle at time $k$ is represented as $\left\{x_{e n u, k}^{(i)}, y_{e n u, k}^{(i)}, v_{v e h, k}^{(i)}, \gamma_{v e h, k}^{(i)}\right\}$, where $\left(x_{e n u, k}^{(i)}, y_{e n u, k}^{(i)}\right)$ is the particle coordinate in ENU space, $v_{v e h, k}^{(i)}$ and $\gamma_{v e h, k}^{(i)}$ are particle velocity and yaw angle respectively. $v_{v e h, k}^{(i)}$ and $\gamma_{v e h, k}^{(i)}$ are determined according to uniform distribution based on vehicle velocity $v_{v e h, k}$ and vehicle yaw angle $\gamma_{v e h, k}$. So the displacement of a single particle is computed as $\triangle L_{e n u, k}^{(i)}=v_{v e h, k}^{(i)} \cdot \Delta T_{p f}$, where $\triangle T_{p f}$ is the time interval of a filter cycle. $\triangle L_{e n u, k}^{(i)}$ is then scaled to BEV space as $\triangle L_{i p m, k}^{(i)}$. The trajectory length along marking model $x=c+d \cdot y+e \cdot y^{2}$ from the starting point $(c, 0)$ to any point on the parabola $(x, y)$ can be expressed as:

$$
\begin{aligned}
L(y)= & \int_{0}^{y} \sqrt{1+(\partial x / \partial y)^{2}} d y \\
= & \frac{1}{2 e}\left\{\frac{d+2 e y}{2} \sqrt{(d+2 e y)^{2}+1}+\frac{1}{2} \ln [(d+2 e y)\right. \\
& \left.+\sqrt{(d+2 e y)^{2}+1}\right]-\frac{d}{2} \sqrt{d^{2}+1} \\
& \left.-\frac{1}{2} \ln \left[d+\sqrt{d^{2}+1}\right]\right\} .
\end{aligned}
$$

Eq. (1) is derived from the integration expression $\int \sqrt{x^{2}+1} d x=\frac{x}{2} \sqrt{x^{2}+1}+\frac{1}{2} \ln \left(x+\sqrt{x^{2}+1}\right)+C$.

Therefore, the relative displacement of a particle is given by

$$
\begin{aligned}
& \triangle y_{i p m, k}^{(i)}=\underset{y \in(0,+\infty)}{\arg \min }\left|\triangle L_{i p m, k}^{(i)}-L(y)\right|, \\
& \triangle x_{i p m, k}^{(i)}=c+d \cdot \triangle y_{i p m, k}^{(i)}+e \cdot\left(\triangle y_{i p m, k}^{(i)}\right)^{2} .
\end{aligned}
$$

$\triangle x_{i p m, k}^{(i)}$ and $\triangle y_{i p m, k}^{(i)}$ are then translated to ENU space. The particle state after movement is then given by:

$$
\begin{aligned}
& x_{e n u, k}^{(i)}=x_{e n u, k-1}^{(i)}+\triangle x_{e n u, k}^{(i)}, \\
& y_{\text {enu }, k}^{(i)}=y_{\text {enu, }, k-1}^{(i)}+\triangle y_{\text {enu }, k}^{(i)} .
\end{aligned}
$$

The normalized importance weight $\bar{w}_{k}^{(i)}$ of a particle is calculated according to a two-dimension Gaussian distribution centering at $\left(x_{e n u, k}, y_{e n u, k}\right)$. The particles are resampled according to Sequential Importance Resampling (SIR) algorithm. Finally, the approximated vehicle fix is given by

$$
\left(\hat{x}_{e n u, k}, \hat{y}_{e n u, k}\right)=\left(\sum_{i=1}^{N_{p f}} \bar{w}_{k}^{(i)} \cdot x_{e n u, k}^{(i)}, \sum_{i=1}^{N_{p f}} \bar{w}_{k}^{(i)} \cdot y_{e n u, k}^{(i)}\right) \text {. }
$$

\section{B. Lane Selection}

The filtered vehicle position is used to select current lane in this step. The aim of lane selection is to derive the map-based lane markings. The markings are obtained from OpenStreetMap (OSM), a collaborative project to provide open source map database. In OSM, the coordinates of road sections are provided, as well as the number of lanes and lane width in each section. With these information, the original node topology can be reconstructed to a lanemarking topology. When the current lane is selected, the current lane markings are derived.

Lane selection stage is implemented in two steps. In the first step, map information and filtered vehicle positions are fused to decide which part of road the vehicle is in. The nearest road section to the filtered vehicle position is regarded as the current road section. A road section may contain one or more lanes. The second step is to determine which lane the vehicle is in in a road section, according to multi-criterion. Considered criteria include lane changing criterion, third lane marking criterion, and history vehicle state criterion. Assuming that the maximum lane number is 3 , which is left lane, middle lane, and right lane. The corresponding probabilities of each lane are $p_{l}, p_{m}$, and $p_{r}$. The first criterion is lane changing, which is determined according to the zero order component $c$ in lane marking model $x=c+d \cdot y+e \cdot y^{2}$. The strategy is that a lane changing from a previous lane to a following lane distributes also the probability of the previous lane, $p_{l, l c}, p_{m, l c}$ and $p_{r, l c}$ are the probabilities of left, middle, and right lane, respectively.

The second criterion is the third lane marking. At first, the third lane marking parameter range is estimated through the detected two current lane markings in Sect II. $p_{l, m k}, p_{m, m k}$ and $p_{r, m k}$ are the probabilities of third lane marking criteria respectively. If a third marking on the left side is detected, the right lane probabilities $p_{m, m k}$ and $p_{r, m k}$ increase.

The third criterion is the historical vehicle state in previous states. The probabilities of historical state are represented as 
$p_{l, h s}, p_{m, h s}$, and $p_{r, h s}$. Therefore, the integrated probabilities of lanes are:

$$
\begin{aligned}
p_{l} & =k_{l c} p_{l, l c}+k_{m k} p_{l, m k}+k_{h s} p_{l, h s}, \\
p_{m} & =k_{l c} p_{m, l c}+k_{m k} p_{m, m k}+k_{h s} p_{m, h s}, \\
p_{r} & =k_{l c} p_{r, l c}+k_{m k} p_{r, m k}+k_{h s} p_{r, h s},
\end{aligned}
$$

where $k_{l c}=0.6, k_{m k}=0.24$ and $k_{h s}=0.16$ are coefficients of the three cues, used to tune the importance of different cues.

The lane whose probability is the maximum value among $p_{l}, p_{m}$ and $p_{r}$ is defined as the lane state $S_{\text {lane }}$ of current lane. The map-based lane markings of current state can be thus derived.

\section{Marking Based Localization}

When both vision-based and map-based lane markings of current lane are obtained, the vehicle position is derived using a multi-kernel based estimation method. Fig. 1 illustrates the vehicle localization procedure. The markings of current cell and the cell in front are projected to BEV space, as the black lines in Fig. 1. The sets of left and right marking pixels are denoted as $S_{l, i p m}$ and $S_{r, i p m}$ respectively. Vision-based lane markings are represented as the form of quadratic model $\left(c_{i}^{*}, d_{i}^{*}, e_{i}^{*}\right), i=l, r$ (see Section II), as the gray curves in Fig. 1. The transformation from $\left(c_{i}^{*}, d_{i}^{*}, e_{i}^{*}\right), i=l, r$ to $S_{l, i p m}$ and $S_{r, i p m}$ can be regarded as motion from rough GPS data to positions on the map.

A transformation matrix from map-based markings to vision-based lane markings is estimated according to a multikernel based estimation method. The translation matrix is represented by $T_{l o c}\left(\triangle x_{l o c}, \triangle \theta_{l o c}\right)$, where $\triangle x_{l o c}$ is lateral displacement, and $\triangle \theta_{l o c}$ is vehicle rotation. A multi-kernel based descriptor $G_{p}\left(c_{i}^{*}, d_{i}^{*}, e_{i}^{*}, x, y\right)$, represented as Eq. (10), is introduced as the "distance" between a single pixel on a map-based marking and a vision-based marking model.

$$
G_{p i}\left(c_{i}^{*}, d_{i}^{*}, e_{i}^{*}, x_{i}, y_{i}\right)=\sum_{y=y_{i}-2 \sigma}^{y_{i}+2 \sigma} K_{x}\left(x_{i}, y_{i}, y\right) K_{y}\left(y_{i}, y\right)
$$

where

$$
\begin{aligned}
K_{y}\left(y_{i}, y\right) & =\frac{1}{\sqrt{2 \pi \sigma^{2}}} \exp \left(-\frac{\left(y-y_{i}\right)^{2}}{2 \sigma_{y i}^{2}}\right) \\
K_{x}\left(x_{i}, y_{i}, y\right) & =\frac{1}{\sqrt{2 \pi \sigma^{2}}} \exp \left(-\frac{\left(c+d y+e y^{2}-x_{i}\right)^{2}}{2 \sigma_{x i}^{2}}\right) .
\end{aligned}
$$

$K_{x}$ and $K_{y}$ are computed according to look-up table. The optimized lateral displacement $\triangle x_{l o c}^{*}$ and rotation $\triangle \theta_{l o c}^{*}$ are obtained with respect to $G_{p}\left(c_{i}^{*}, d_{i}^{*}, e_{i}^{*}, x, y\right)$ as

$$
\left(\triangle x_{l o c}^{*}, \triangle \theta_{l o c}^{*}\right)=\underset{\triangle x_{l o c}, \triangle \theta_{l o}}{\arg \max } \sum_{\substack{(\mathrm{x}, \mathrm{y}) \\ \mathrm{S}_{i, t r a}}}^{r} G_{p}\left(c_{i}^{*}, d_{i}^{*}, e_{i}^{*}, x, y\right),
$$

where

$S_{i, t r a}=\left\{(x, y) \mid(x, y, 1)=\left(x^{\prime}, y^{\prime}, 1\right) \cdot T_{l o c},\left(x^{\prime}, y^{\prime}\right) \in S_{i, i p m}\right\}$

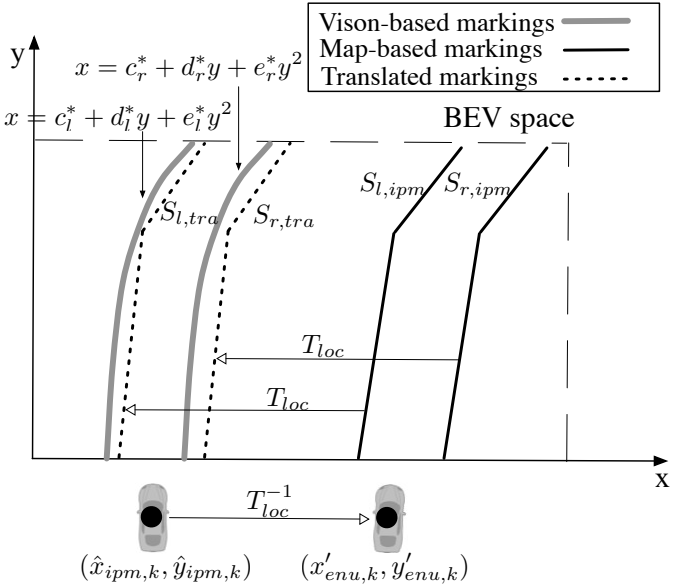

Fig. 1. Vehicle localization procedure.

$S_{i, i p m}$ is the set of lane marking pixels in BEV space, $S_{i, t r a}$ is the projected pixel set. Translated markings through $T_{l o c}\left(\triangle x_{l o c}^{*}, \triangle \theta_{l o c}^{*}\right)$ are illustrated as dashed lines in Fig. 1. The inverse matrix $T_{l o c}^{-1}\left(\triangle x_{l o c}^{*}, \triangle \theta_{l o c}^{*}\right)$ of $T_{l o c}\left(\triangle x_{l o c}^{*}, \triangle \theta_{\text {loc }}^{*}\right)$ is the correction transformation of lane marking based vehicle localization. So $\left(x_{i p m, k}^{\prime}, y_{i p m, k}^{\prime}\right)$ is computed as

$\left(x_{i p m, k}^{\prime}, y_{i p m, k}^{\prime}, 1\right)=\left(\hat{x}_{i p m, k}, \hat{y}_{i p m, k}, 1\right) \cdot T_{l o c}^{-1}\left(\triangle x_{l o c}^{*}, \triangle \theta_{l o c}^{*}\right)$.

The marking based localized position is $\left(x_{e n u, k}^{\prime}, y_{e n u, k}^{\prime}\right)$, through the transfer from BEV space to ENU space.

\section{PROBABILISTIC ERROR MODELING}

In order to estimate the confidence of the vehicle localization procedure, a representation of potential error sources in localization method can be identified. To this end, a probabilistic error modeling is proposed. Normally, the main problems which cause unsuccessful localization are errors of lane detection, errors of vehicle localization, and errors of map topology. The models of these errors can be constructed lying on several criteria, such as marking detection cues, third marking cues, and localization fitting cues.

\section{A. Error Modeling}

The confidences of lane detection error model, localization error model and map error model are estimated. The potential errors are decided according to methods based on the following models.

Error model of lane detection. Improper vision-based marking detection leads to a dismatch with map-based lane marking, and thus, an inaccurate localization. This kind of error can be detected through lane detection confidence con $f_{l d}$. When a marking is detected from an image, con $f_{l d}$ is employed to quantify the quality of a marking, which can be a meaningful criterion to detect when lane detection fails. To this end, a set $\Phi_{c}$ of pixels with higher contribution to the detected markings $\left(c^{*}, d^{*}, e^{*}\right)$ are defined as follows:

$$
\Phi_{c}=\left\{\left(x_{i}, y_{i}\right) \mid G_{p i}\left(c^{*}, d^{*}, e^{*}, x_{i}, y_{i}\right) \geq G_{\text {thres }}\right\},
$$


where $i=1, \ldots, n_{\text {con }}, G_{\text {thres }}$ is confidence threshold, and $n_{c o n}$ is the total number of high contributed pixels in the image.

Then the image is divided into $L$ blocks with the same length along y-axis of BEV coordinate. The set $\Phi_{c}^{l}$ of pixels in $l^{\text {th }}$ block is defined as:

$\Phi_{c}^{l}=\left\{\left(x_{i}, y_{i}\right) \mid\left(x_{i}, y_{i}\right) \in \Phi_{c}, \frac{M}{L} l \leq y_{i}<\frac{M}{L}(l+1)\right\}$,

where $l=0,1, \ldots, L-1, i=1, \ldots, n_{\text {con }, l}, n_{\text {con }, l}$ is the number of high contributed pixels in $l^{\text {th }}$ block.

When the number of high contributed pixels in all the blocks are determined, the confidence of a detection can be estimated as:

$$
\operatorname{conf}_{l d}=\frac{n_{c o n}}{L} \sum_{l=0}^{L-1} p_{c o n, l}
$$

where

$$
p_{c o n, l}=\left\{\begin{array}{ll}
\frac{L \cdot n_{\text {con }, i}}{n_{\text {con }}} & \left(0 \leq n_{\text {con }, i}<\frac{n_{c o n}}{L}\right) \\
\frac{L \cdot n_{c o n, i}}{(1-L) \cdot n_{\text {con }}}+\frac{L}{L-1}\left(\frac{n_{c o n}}{L} \leq n_{\text {con }, i}<n_{c o n}\right)
\end{array} .\right.
$$

It is worth to mention that conf $f_{l d}$, takes the number of contributed pixels $\left(n_{\text {con }} / L\right.$ in Eq. (18) ) and the distribution of these pixels $\left(\sum_{l=0}^{L-1} p_{c o n, l}\right.$ in Eq. (18) ) into account. A detection result which has more contributed pixels and whose contributed pixels are more equally distributed earns a higher confidence.

A lane detection error is modeled based on con $f_{l d}$ and lane width. An unsuccessful detection contains two conditions. One condition is that confld is lower than a threshold $\overline{\operatorname{conf} f_{l d}}$, as shown in Fig. 3(a). The second condition relies on the differences between the vision-based lane width (blue curves in Fig. 3(b)) and the lane width provided by OSM (red curves in Fig. 3(b)). When both conditions are satisfied, a lane detection error is found, as shown in Fig. 3(c).

Error model of localization. In fitting step of localization (see Section III-C), dismatching situations may happen. For instance, the vision-based left marking matches the mapbased right marking, rather than map-based left marking. Marking fitting confidence $\operatorname{conf}_{f t}$ is used to detect this kind of failure. When $\triangle x_{l o c}^{*}$ and $\triangle \theta_{l o c}^{*}$ are computed, error confidence is defined according to Eq. (13):

$\operatorname{conf}_{f t}=\frac{\left|\sum_{(x, y) \in S_{l, t r a}} G_{p}(x, y)-\sum_{(x, y) \in S_{r, t r a}} G_{p}(x, y)\right|}{\sum_{(x, y) \in S_{l, t r a}} G_{p}(x, y)+\sum_{(x, y) \in S_{r, t r a}} G_{p}(x, y)}$

When $\operatorname{conf}_{f t}$ tends to 0 , it implies a high quality marking fitting, because the left and right fitting confidence $\sum_{(x, y) \in S_{l, t r a}} G_{p}(x, y)$ and $\sum_{(x, y) \in S_{r, t r a}} G_{p}(x, y)$ are almost equal. If $\operatorname{con} f_{f t}$ is higher than threshold $\overline{\operatorname{conf} f t}$, a localization failure is identified. This procedure is demonstrated in Fig. 4.

Error model of map topology. A map error here stands for a fault lane number assigned to a road segment in OSM. For instance, the host vehicle is in a two-lane section, but the lane number offered from OSM is 3. Third lane markings, mentioned in Section III-B, can be used to identify this
TABLE I

\begin{tabular}{|c|c|c|c|}
\hline & \multicolumn{2}{|c|}{ with threshold $\overline{\operatorname{conf} f_{l d}}$} \\
\hline & & $\overline{\operatorname{con} f_{l d} \leq \overline{\operatorname{con} f_{l d}}}$ & $\operatorname{con} f_{l d}>\overline{\operatorname{con} f_{l d}}$ \\
\hline \multirow{2}{*}{$\begin{array}{c}\text { with } \\
\text { ground truth }\end{array}$} & an error & $\mathrm{TP}$ & FN \\
\hline & not an error & FP & TN \\
\hline
\end{tabular}

CONFUSION MATRIX OF con $f_{l d}$

TABLE II

\begin{tabular}{|c|c|c|c|}
\hline & \multicolumn{2}{|c|}{ with threshold $\overline{c o n f_{f t}}$} \\
\hline & & $\overline{\operatorname{con} f_{f t} \leq \overline{\operatorname{con} f_{f t}}}$ & $\operatorname{con} f_{f t}>\overline{\operatorname{con} f_{f t}}$ \\
\hline \multirow{2}{*}{$\begin{array}{c}\text { with } \\
\text { ground truth }\end{array}$} & an error & $\mathrm{FN}$ & $\mathrm{TP}$ \\
\hline & not an error & $\mathrm{TN}$ & FP \\
\hline
\end{tabular}

CONFUSION MATRIX OF con $f_{f t}$

error. The third marking confidence conf $f_{\text {trd }}$ represents the probability of the lane markings to form the neighboring lanes. When third markings are derived, marking confidence conf $f_{t r d}$ can be calculated by the same method in Eq. (18). The third lane markings with confidence $c o n f_{t r d}$ greater than $\overline{\operatorname{conf} f_{\text {trd }}}$ are considered as potential third markings, shown as green markers in Fig. 5(b) and (c). Barriers or other noises sometimes can cause an instantaneous spourious third marking, so the markings keeping visible for a period are considered as a detected third marking, as red markers in Fig. 5(b) and (c). Once the third lane markings are derived, map error can be modeled logically through fusing third marking and number of lanes from OSM (red curves in 5(a)). For instance, when the vision detects a third lane marking, but the map database indicates the current section is one-lane road section, an error is identified.

\section{B. Threshold Estimation}

When the confidences confld and conf $f_{f t}$ are estimated, the potential error can be inferred according to the thresholds $\overline{\operatorname{conf} f_{l d}}$ and $\overline{\operatorname{conf} f_{f t}}$. To determine the optimized thresholds, confusion matrices of confld and conf $f_{f t}$ are constructed as Table I and II respectively, including the required confusion matrix elements: true positive (TP), false positive (FP), true negative $(\mathrm{TN})$ or false negative (FN).

A higher confidence con $f_{l d}$ means a lower possibility of lane detection error, while a higher confidence $\operatorname{con} f_{f t}$ represents a higher possibility of localization error. Therefore, the definition of matrix elements in Table I and II are different. The optimized thresholds are derived using ROC curve [18]. The thresholds are valued as $\overline{\operatorname{conf}_{l d}}=400, \overline{\text { conf }_{f t}}=0.8$. The third marking threshold $\overline{\operatorname{conf} f_{\text {trd }}}$ is assigned the same as marking detection threshold $\overline{\operatorname{conf} f_{l d}}$.

\section{RESULTS}

Our method has been evaluated on KITTI database [19]. The input images are captured by a facing-front gray-scale camera mounted on the vehicle. The raw GPS signals are produced by adding noise model on high precision GPS positions provided by KITTI. This method is implemented using $\mathrm{C}++$, and the obtained result data are analyzed using Matlab.

\section{A. Localization Results}

The results of marking based localization are depicted in Fig. 2. Fig. 2(a)-(b) are two zoomed map areas. In Fig. 2(a), the pink curve represents the vehicle positions filtered from 


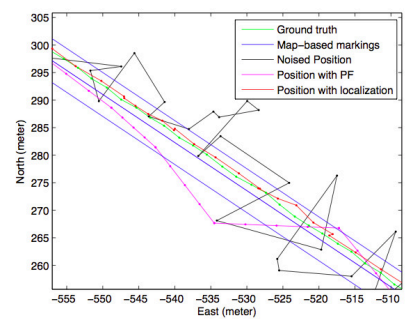

(a)

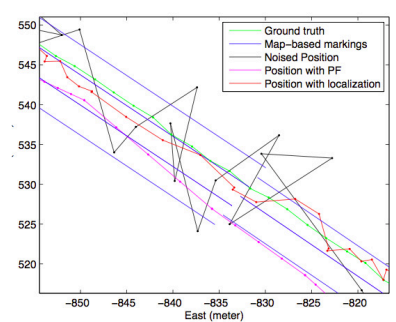

(b)
Fig. 2. Localization result.

TABLE III

ERROR STATISTICS.

\begin{tabular}{|c|c|c|}
\hline & $\begin{array}{c}\text { Lateral position error } \\
\text { after particle filter }\end{array}$ & $\begin{array}{c}\text { Lateral position error } \\
\text { after localization }\end{array}$ \\
\hline Mean value & $1.884 \mathrm{~m}$ & $0.089 \mathrm{~m}$ \\
\hline MAE & $2.867 \mathrm{~m}$ & $1.006 \mathrm{~m}$ \\
\hline Standard deviation & $2.942 \mathrm{~m}$ & $1.284 \mathrm{~m}$ \\
\hline Max & $9.083 \mathrm{~m}$ & $5.429 \mathrm{~m}$ \\
\hline 95th percentile & $6.345 \mathrm{~m}$ & $2.589 \mathrm{~m}$ \\
\hline
\end{tabular}

rough GPS signals (black), using particle filter. However, this pink curve is not in the current road section. The vehicle positions on pink curve are used to select current road lane according to multi-criterion. When the road lane is determined, marking based localization is implemented, the vehicle position is adjusted to the central area of road, as red curve in Fig. 2(a). Fig. 2(b) depicts an example: a mismatch occurred between two sources, benchmark positions from KITTI and map information from OSM. In this example, the ground truth GPS data is in the middle of two lanes, but in the vision, the vehicle is in the middle lane. One can infer that at least one source is not accurate. Such mismatch leads to an error even if the marking based method is utilized. The localization result of the entire scenario is shown in Fig. 6(a).

Table III provides the performance metrics of localization results. In Table III, the mean value of both errors are far below the noise error bound $(14.2 \mathrm{~m})$. The maximum error of particle filter $(9.083 \mathrm{~m})$ does not exceed the noise error bound neither. Comparing position errors of the two methods, all the statistics of marking based method are less than that of particle filter, which numerically proves that marking based localization helps to improve the performance of vehicle fix.

\section{B. Error Modeling Results}

The identified errors are compared with error ground truth, which were annotated manually frame by frame. The observed lane detection error with ground truth is illustrated in Fig. 3(c). In frame 1080, a lane detection error is detected, because the lane marking confidence $c o n f_{l d}$ is lower than the threshold and the lane width is abnormal. Such kind of false alarm can be caused by an affected lane marker or a poor vision.

The error state of vehicle localization is shown in Fig. (4). False alarm states happen in frame 550, an unusual frame is detected where no error occurred actually. The reason is that the lane widths from map source and from vision detection are different, leading to a difficulty in marking fitting.

The map errors are depicted in Fig. 5(a), as well as the ground truth. In frame 980, the lane number of the current
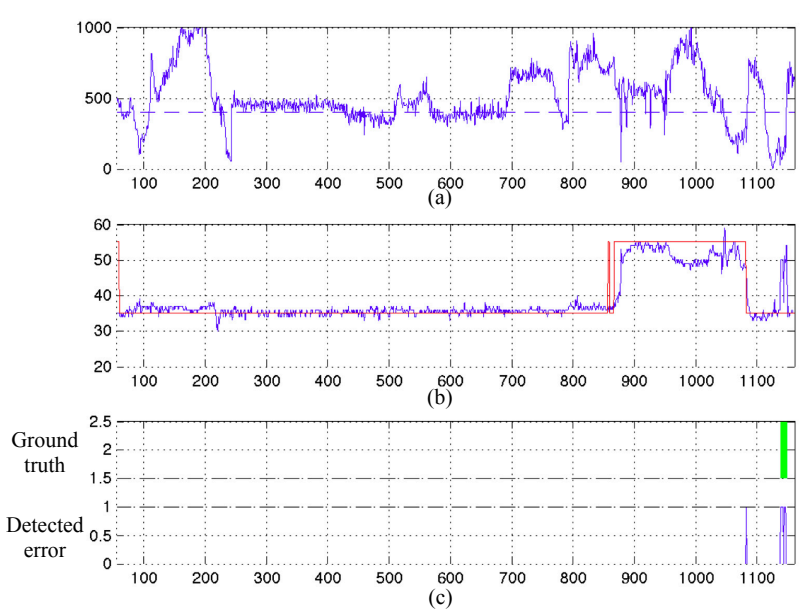

Fig. 3. The lane detection error. (a) marking confidence, (b) detected lane width and map-based lane width, (c) detected error and ground truth.

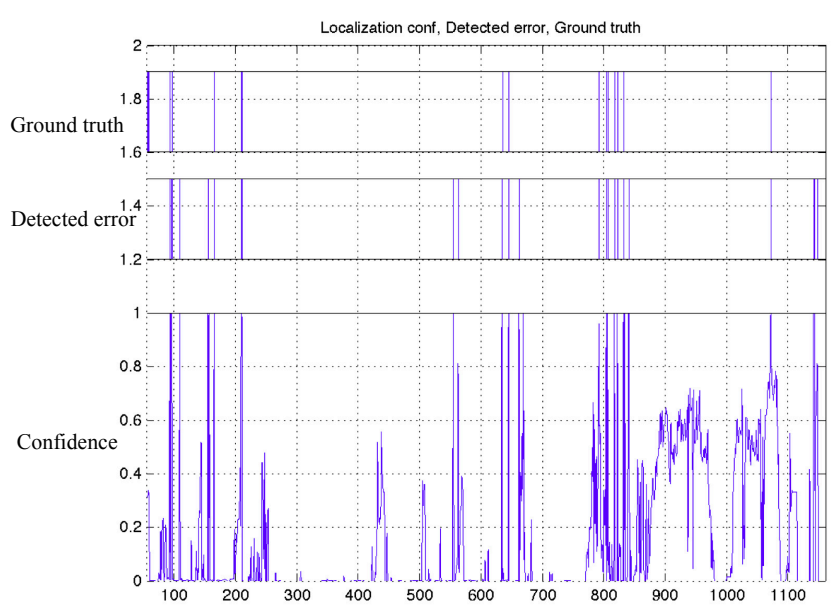

Fig. 4. Localization error.

section is one, while from vision, the vehicle is in a twolane road section. A third lane marking helps to catch this error. From frame 1100 to frame 1120, the map error frames do not seem fit strictly, because the vision has discovered a third lane marking in the following two-lane section, but the vehicle itself is still in the previous one-lane section. In frame 1040, the barrier is detected as a third lane marking by mistake, this instantaneous third marking causes false alarm of map error.

Fig. 6 shows the localization result with the inferred errors. In Fig. 6(a), an original localization result is represented. Blue lines are noise bounds. The cyan curve is the noised GPS position. The position processed by Particle Filter is shown as yellow curve. And the red curve is the optimized vehicle position. The Mean Absolute Error (MAE) of the optimized position is $M A E_{a}=1.006 \mathrm{~m}$. In Fig. 6(b), the ground truth errors in localization method are marked on localization result. The blue columns represent localization errors, green ones are lane detection errors, and pink are map errors. These errors lead to unsuccessful vehicle localization. If these error frames are ignored, the MAE decreases to 

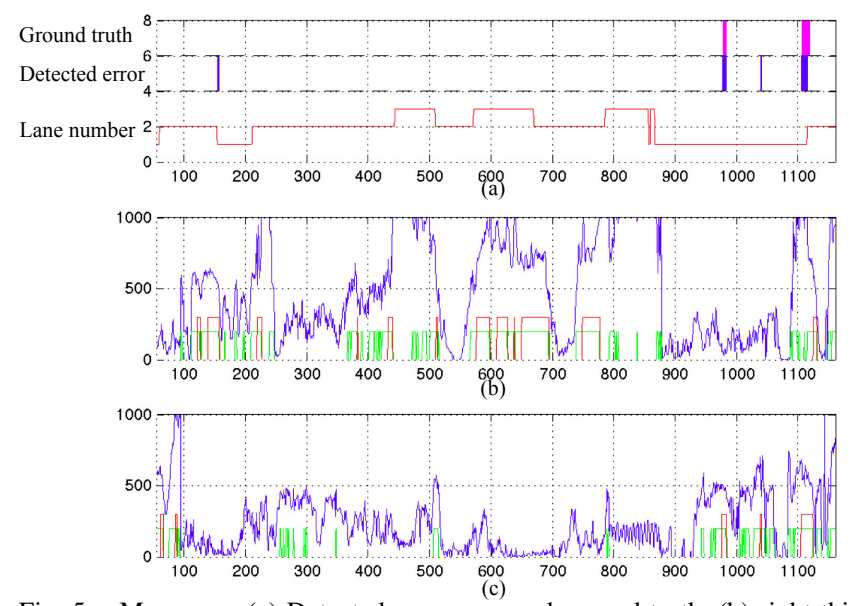

Fig. 5. Map error. (a) Detected map error and ground truth, (b) right third marking confidence, (c) left third marking confidence.
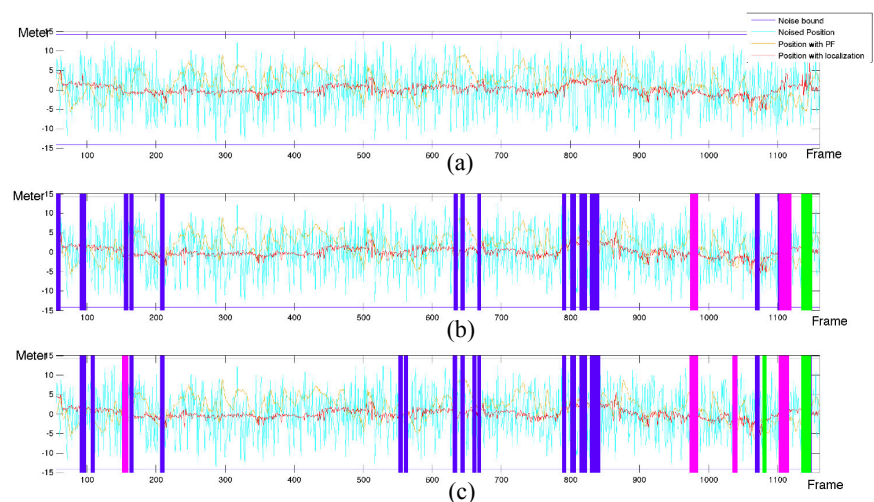

Fig. 6. The localization results and detected errors. (a) Localization result of all the frames in a scenario, (b) localization result with ground truth errors, (c) localization result with identified errors.

$M A E_{b}=0.860 m$. Fig. 6(c) is the same localization result, but with the errors identified by error detection models mentioned in Section IV. If the error frames are removed, the MAE of lateral vehicle position error is $M A E_{c}=0.925 \mathrm{~m}$. $M A E_{c}$ is greater than the $M A E_{b}$, this is because not all the detected errors match ground truth errors. In this experimental scenario, 73 frames are measured as questionable frames but 21 frames of them are not included in ground truth, which means that 52 errors among a total number of 73 are detected correctly, the success ratio is $71.23 \%$. Meanwhile, 13 error frames are not identified by the error modeling and thresholding. These false alarm errors and undetected errors causes a larger MAE in Fig. 6(c) than (b), which is $M A E_{c}>M A E_{b}$. However, $M A E_{c}$ is lower than $M A E_{a}$, which means that the probabilistic error modeling helps to improve vehicle localization performance.

\section{CONCLUSIONS}

A lane marking based vehicle localization, using open source map dataset and low-cost sensors has been demonstrated. The results verify a real-time and precise vehicle localization. An enhanced multi-kernel based method is represented to detect lane markings and optimize vehicle positions. The vehicle position is adjusted in two steps. Firstly, a particle filter is designed to adjust the rough GPS position. Secondly, vision-based markings and map-based markings are fused to obtain vehicle position. Corresponding potential errors are identified according to probabilistic error models.

Future work will focus on how to cope with different errors into error detection algorithm. Meanwhile, map reconfiguration and road connections and road branches are needed to be improved.

\section{REFERENCES}

[1] J. Bentley and H. Maurer, "Efficient worst-case data structures for range searching," Acta Informatica, vol. 13, pp. 155-168, 1980.

[2] C. E. White, D. Bernstein, and A. L. Kornhauser, "Some map matching algorithms for personal navigation assistants," Transportation Research Part C: Emerging Technologies, vol. 8, no. 1-6, pp. 91-108, FebruaryDecember 2000.

[3] B. P. Phuyal, "Method and use of aggregated dead reckoning sensor and gps data for map matching," in Proceedings of the 15th International Technical Meeting of the Satellite Division of The Institute of Navigation, September 2002, pp. 430-437.

[4] Y. Meng, "Improved positioning of land vehicle in its using digital map and other accessory information," Ph.D. dissertation, Hong Kong Polytechnic University, 2006.

[5] M. A. Quddusa, W. Y. Ochiengb, and R. B. Noland, "Current mapmatching algorithms for transport applications: State-of-the art and future research directions," Transportation Research Part C: Emerging Technologies, vol. 15, no. 5, pp. 312-328, October 2007.

[6] W. Y. Ochieng, M. A. Quddus, and R. B. Noland, "Map-matching in complex urban road networks," Brazilian Society of Cartography, Geodesy, Photogrammetry and Remote Sensing (SBC), 2003.

[7] N. Mattern, R. Schubert, and G. Wanielik, "High-accurate vehicle localization using digital maps and coherency images," in IEEE Intelligent Vehicles Symposium, June 2010, pp. 462-469.

[8] A. Barth, J. Siegemund, and J. Schwehr, "Fast and precise localization at stop intersections," in IEEE Intelligent Vehicles Symposium Workshops, June 2013, pp. 75-80.

[9] Z. Tao, P. Bonnifait, V. Frémont, and J. Ibañez-Guzman, "Mapping and localization using gps, lane markings and proprioceptive," in IEEE/RSJ Conf. on Intelligent Robots and Systems, November 2013, pp. 406-412.

[10] H. Lategahn, M. Schreiber, J. Ziegler, and C. Stiller, "Urban localization with camera and inertial measurement unit," in IEEE Intelligent Vehicles Symposium, June 2013, pp. 719-724.

[11] E. Seignez, M. Kieffer, A. Lambert, E. Walter, and T. Maurin, "Real-time bounded-error state estimation for vehicle tracking," The International Journal of Robotics Research, vol. 28, no. 1, pp. 34-48, January 2009.

[12] S. A. Rodríguez F, V. Fremont, P. Bonnifait, and V. Cherfaoui, "An embedded multi-modal system for object localization and tracking," IEEE Intelligent Transportation Systems Magazine, vol. 4, no. 4, pp. 42-53, November 2012.

[13] T. Wu and A. Ranganathan, "Vehicle localization using road markings," in IEEE Intelligent Vehicles Symposium, June 2013, pp. 1185-1190.

[14] D. Gruyer, R. Belaroussi, and M. Revilloud, "Map-aided localization with lateral perception," in IEEE Intelligent Vehicles Symposium, June 2014, pp. 674-680.

[15] Z. Tao, P. Bonnifait, and V. Frémont, "Lane marking aided vehicle localization," in IEEE Conf. on Intelligent Transportation Systems, November 2013.

[16] W. Lu, S. Rodriguez, E. Seignez, and R. Reynaud, "Monocular multikernel based lane marking detection," in IEEE 4th Annual International Conference on Cyber Technology in Automation, Control, and Intelligent Systems (CYBER), June 2014.

[17] R. Jiang, R. Klette, T. Vaudrey, and S. Wang, "Lane detection and tracking using a new lane model and distance transform," Machine Vision and Applications, vol. 22, no. 4, pp. 721-737, June 2011.

[18] T. Fawcett, "An introduction to roc analysis," Pattern Recognition Letters, vol. 27, no. 8, pp. 861-874, June 2006.

[19] A. Geiger, P. Lenz, C. Stiller, and R. Urtasun, "Vision meets robotics: The kitti dataset," International Journal of Robotics Research, vol. 32, no. 11, pp. 1231-1237, September 2013. 